\title{
SPECTROSCOPIC OBSERVATIONS OF SOLAR SYSTEM OBJECTS: PUSHING THE LIMITS
}

\author{
Anita L. Cochran \\ The University of Texas, McDonald Observatory
}

\section{INTRODUCTION}

Targets within the solar system generally fall into one of two types: a) major planets (except Pluto) and our Moon; b) minor planets, comets, Pluto and planetary satellites. The first group is noteworthy for being reasonably bright. Most are also spatially extended. The inner planets never achieve large solar elongation. The second group comprises bodies which are generally faint. Comets are spatially extended. The minor planets and comets may be in orbits which are highly inclined or viewed at small solar elongations. Comets may even be in retrograde orbits. Planetary satellites may be bright or faint but suffer from being in the glare of the parent planet.

As with most aspects of astronomy, the study of bodies within our solar system has seen rapid progress with the advent of modern array detectors. Array detectors assist by having good quantum efficiency, facilitating faster observations, increased spectral coverage over plates or photomultipliers, high signal/noise ratio allowing detection of low contrast features, large dynamic range, and spatial coverage which is useful for extended objects or to remove a sloping background.

Much success in solar system observations have come about in the IR. However, that is beyond the scope of this report. In this paper, I will confine myself to the optical region of the spectrum and demonstrate the remarkable advances of the past decade or so which have been made possible through the use of modern array detectors.

\section{MAJOR PLANETS: JUPITER}

Jupiter is one of the brightest objects in the sky and thus, it would seem to be a simple body to observe. It is spatially extended, reaching a diameter of $45 \operatorname{arcsec}$ at opposition. It is an interesting object to study since it has a dynamically changing atmosphere. However, with a rotation period of $9^{\mathrm{h}} 56^{\mathrm{m}}$, spectra must be obtained rapidly to avoid spatial smearing.

The spectrum of Jupiter is dominated by molecular features. Many of these molecules, such as $\mathrm{CH}_{4}$ or $\mathrm{NH}_{3}$, have dense absorption bands. Thus, it takes reasonably high spectral resolution to resolve the structure of the bands. Some of these molecules are strong absorbers, blocking out views of the underlying planet. At other wavelengths, Jupiter is quite bright. Thus, dynamic range becomes quite important to obtain good spectral data. 
The state of the art for observations in the mid-1970s was exemplified by the work of Woodman et al. (1979). In their Fig. 1, they show a spectrum of the central meridian of the equatorial region of Jupiter obtained with a coudé scanner on the $2.7-\mathrm{m}$ telescope at McDonald Observatory. The spectrum covers the spectral range from $6000-10,800 \AA$ with a resolving power $(R)$ of 500 . The exposure had a duration of seven min.

Inspection of their Fig. 1 shows a high signal/noise ratio spectrum with broad molecular emissions. The $8900 \AA \mathrm{CH}_{4}$ band reaches to about $10 \%$ residual intensity. There is an $\mathrm{NH}_{3}$ feature at $6450 \AA$ which shows as a double-lobed absorption.

In contrast, Fig. 1 shows a spectrum obtained by W. Cochran and K. Baines using a Cassegrain echelle spectrograph on the McDonald Observatory 2.1-m telescope in April 1993. This spectrum looks, at first glance, to have quite a low signal/noise ratio. However, that is a false impression due to the compressed spectral scale. These data were obtained with $\mathrm{R}=$ 60,000 using a cross-dispersed echelle imaged onto a Reticon CCD detector. The spectrum in the upper panel contains the complete 31 orders. Terrestrial and solar lines have been removed. The exposure time was two min.

The bottom panel of Fig. 1 shows the same $6450 \AA \mathrm{NH}_{3}$ band observed with the Cassegrain echelle which was described above in the discussion of the Woodman et al. (1979) data. In the expanded view, it is obvious that the molecular band is not composed of only two parts but of many individual lines. The noise level of this spectrum can be estimated from looking at the continuum in the bottom panel.

Certainly, this spectrum has a much higher information content than the spectra of Woodman et al. (1979). The higher signal/noise ratio was made possible by the use of a modern array detector. The CCD allows for the inclusion of many orders from the cross-dispersed echelle; it has more sensitivity allowing for much higher spectral resolution in one third the time and has excellent dynamic range to allow for the strong absorbers. However, it does have only one half the spectral coverage of the scanner data, so two spectra will be needed to cover the same spectral range.

More recently, there is a cross-dispersed echelle spectrograph, 2DCoudé, for the $2.7-\mathrm{m}$ telescope which allows 59 echelle orders to be imaged onto a Tektronixs 2048 x 2048 pixel $^{2}$ CCD with $R=60,000$. This then yields a spectrum of Jupiter in three min with spectral coverage from 0.4 to $1.0 \mu \mathrm{m}$.

\section{THE MOON}

The Moon is perhaps the most familiar of all solar system bodies. Its brightness and size, along with its changing phases have made it an object of notice, study and contemplation since the start of time. Its nearness has also made it a target for exploration.

With the Apollo missions to the Moon, we were able to perform studies of this body which are unrivaled on other extraterrestrial bodies. Samples were returned to the lab and in situ observations were performed. Despite such measurements, at the end of the Apollo missions, we knew that the Moon had an atmosphere (correctly an exosphere since gas-phase collisions are negligible) with a pressure over the subsolar point of $10^{6}$ atoms $\mathrm{cm}^{-3}$. However, 


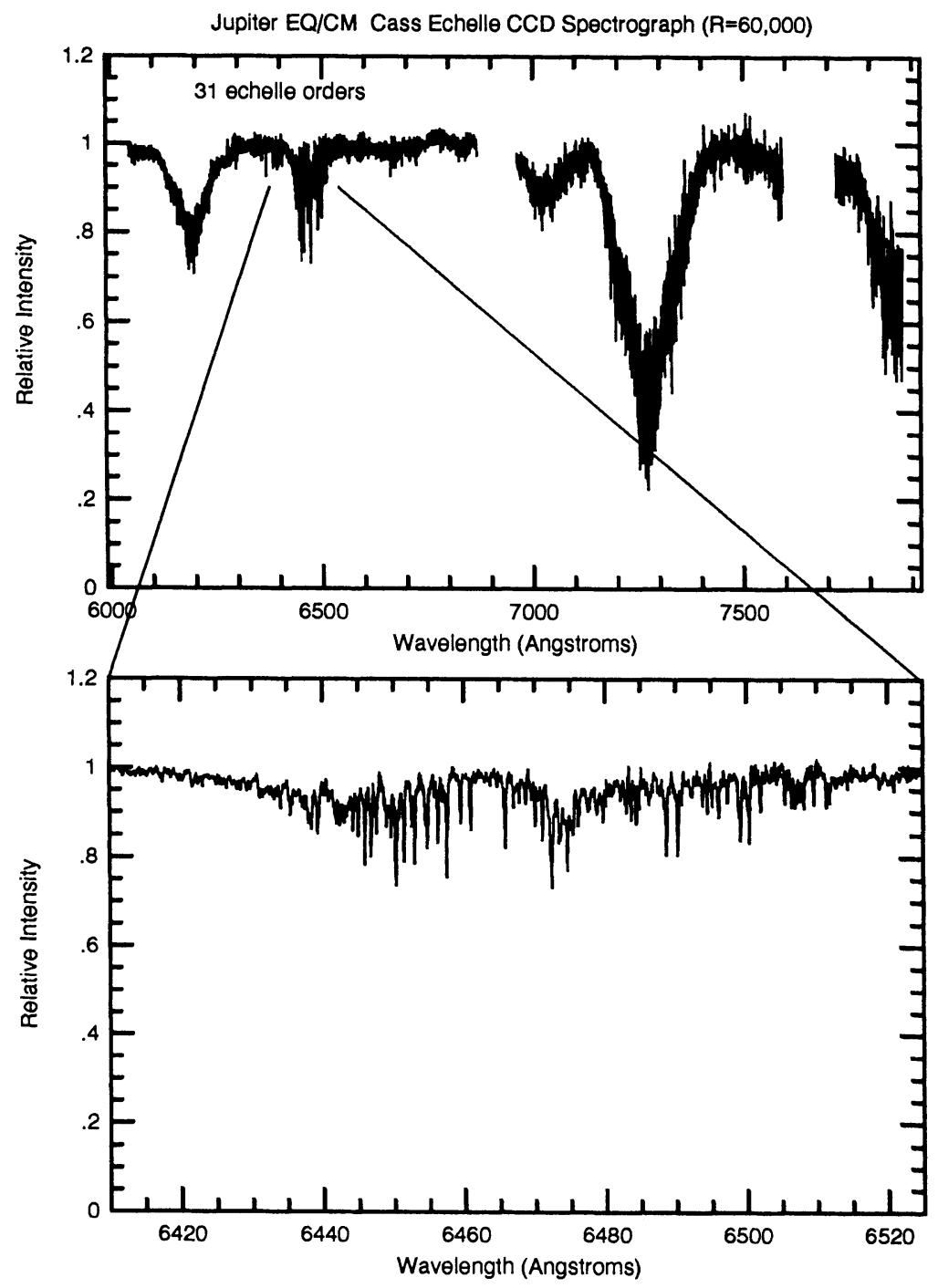

Fig. 1. A spectrum of Jupiter. This spectrum of Jupiter was obtained with a Cassegrain echelle spectrograph on the $2.1-\mathrm{m}$ telescope at McDonald Observatory. The upper panel shows the complete 31 echelle orders obtained with $\mathrm{R}=60,000$. This exposure time is two min. The lower panel shows a spectral blowup of the region around the $6450 \AA \mathrm{NH}_{3}$ band. It is obvious from this spectrum that the signal/noise is high and that the NH3 band is composed of many lines. This spectrum is courtesy of W. Cochran and $\mathrm{K}$. Baines. 
despite our best efforts, the composition of this atmosphere was unknown at the end of the Apollo missions.

Potter and Morgan (1988) first reported positive evidence for atmospheric constituents on the Moon when they detected sodium and potassium above the limb. The atmosphere is quite tenuous and it required the use of very high spectral resolution $(22-100 \mathrm{~m} \AA)$ observations which also contained spatial information to detect the signal against the bright lunar surface. These observations could only be performed with high signal/noise data since the emissions which they detected are found in the cores of the strong solar absorption lines and the Doppler shifts of the solar and lunar spectra are insufficient to move these features away from the solar absorption. Also, an array detector allowed them to obtain spatial information simultaneously with the spectral information to model the scale height of the gas accurately. These observations are a wonderful example of how, even with a very bright source, some questions can only be answered with modern array detectors.

\section{PLANETARY SATELLITES: GALILEAN SATELLITES}

The observations of planetary satellites offer some unique problems which are well handled by modern array detectors. Although many planetary satellites are bright, the brightest satellites tend to be in orbits which are quite near the parent planet. Thus, the background of the satellite can show a strong gradient due to scattered planet light. In the past, this gradient was handled by observing at apoapsis, limiting the phase at which a satellite could be observed, or by separate observations of sky background, either at a symmetric distance from the planet or at a later time. Neither of these approaches allows for observations of the highest quality.

With the advent of current high quality array detectors, observations are once more being obtained of objects such as the Galilean satellites, with interesting new results. Spencer et al. (1994) have used a long slit CCD spectrograph at $18 \AA$ resolution to obtain spectra of all four Galilean satellites from $3200-7800 \AA$. Each spectrum is the sum of $20-30$ spectra of one to two sec each. A comparison solar analogue star was observed with the same instrumental set-up.

Exçept for Io, not many spectra have been obtained of the Galilean satellites since the work of McFadden et al. (1980). The spectra of McFadden et al. (1980) were comprised of data points spaced about $200 \AA$ apart. In these spectra, Europa, Ganymede and Callisto appeared to have much the same reflectance curves. In contrast, the higher signal/noise spectra of Spencer et al. (1994) show that Ganymede and Callisto have similar spectral reflectances but Europa has a strikingly different spectral reflectance.

The high signal/noise of the Spencer et al. (1984) spectra showed what appeared to be a weak absorption feature in the spectrum of Ganymede. Using spectra at $6 \AA$ resolution, Spencer et al. (1984) confirmed the presence of two absorption features of approximately $1 \%$ depth in the spectra of Ganymede which were not present in the spectra of Callisto or Europa. They attribute this absorption feature to solid $\mathrm{O}_{2}$. This interesting discovery could only have been possible with extremely high signal/noise spectra obtained with modern detectors.

\section{MINOR PLANETS}

Perhaps the most progress which has been made in the field of solar system research has 
come with new observations of faint objects. The minor planets constitute a large class of objects which are relatively small bodies with low albedos (3-25\%). The minor planets are interesting because of their surfaces. Spectra show the signature of mineralogy and lattice structure. These features are generally broad and often shallow. Details on the band centers and depths differentiate between various minerals and phases of the same mineral.

Most of the asteroids are so small that they are irregularly shaped bodies. They may have albedo features on the surface which cause changes in the spectrum. Therefore, it is necessary to obtain spectral information as a function of rotational phase. However, many of the rotation periods are short (five to ten hours) so that it is necessary to obtain the spectra quickly, despite the fact that the objects are faint. In this effort, high quantum efficiency is essential.

In addition to rotational phase effects, there are also orbital phase effects. For main-belt asteroids, phase angle variations are generally small. However, for earth-approaching asteroids, phase angle can change rapidly and the window-of-opportunity of observations is short.

It was not until the late 1970s that observers started to obtain more than UBV photometry on asteroids. Still, UBV photometry played a major role in early classification schemes (Chapman et al. 1975). More recently, several multi-color photometric systems have been developed to determine taxonomic differences of asteroids (Chapman and Gaffey 1979 (26 Color Survey); Tholen 1984 (Eight Color Asteroid Survey = ECAS); Bell et al. 1988 (52 Color Survey)).

Today, several groups have started spectral surveys with CCDs which are taking advantage of the higher spectral resolution and excellent quantum efficiency to enhance our understanding of asteroid spectra. An example, from Sawyer (1991), is shown in Fig. 2. In this figure, I show a comparison of four asteroids which were observed with a CCD spectrograph plotted along with results from the ECAS, 26-color and 52-color surveys, as available. These asteroids are of a variety of types and moderately bright. Inspection of Fig. 2 shows that the agreement between the photometry and spectroscopy for 57 Mnemosyne is quite good and the spectroscopy only yields a little more information. For 41 Daphne, the spectroscopy is able to define better the center and shape of the band at $7000 \AA$. Clearly, for 65 Cybele and 93 Minerva, the spectroscopy yields information which is not able to be interpreted in the photometry. Vilas and McFadden (1992), have obtained similar mineralogical information from their spectra.

Recently, Xu et al. (1994) have started their Small Main-Belt Asteroid Spectral Survey (SMASS) to extend the classification of asteroids in the belt to smaller sizes. They use a CCD detector on a Cassegrain spectrograph. Whereas the ECAS survey was limited to objects of $>$ $20 \mathrm{~km}$ in the inner belt and larger than $>55 \mathrm{~km}$ in the outer belt, the SMASS observations have extended to $<5 \mathrm{~km}$ in the inner belt and $<25 \mathrm{~km}$ in the outer belt. At the same time as SMASS observations have been able probe to smaller objects, they have equaled or bettered the signal/noise of all but the best ECAS observations, and with much higher spectral resolution. With this survey, Binzel and Xu (1993) showed that many asteroids existed which may be collisional fragments of Vesta and the source for basaltic achondritic meteorites. In addition Binzel et al. (1993) have discovered an asteroid having a reflectance spectrum very similar to L6 and LL6 ordinary chondrite meteorites. This discovery is significant since the L6 

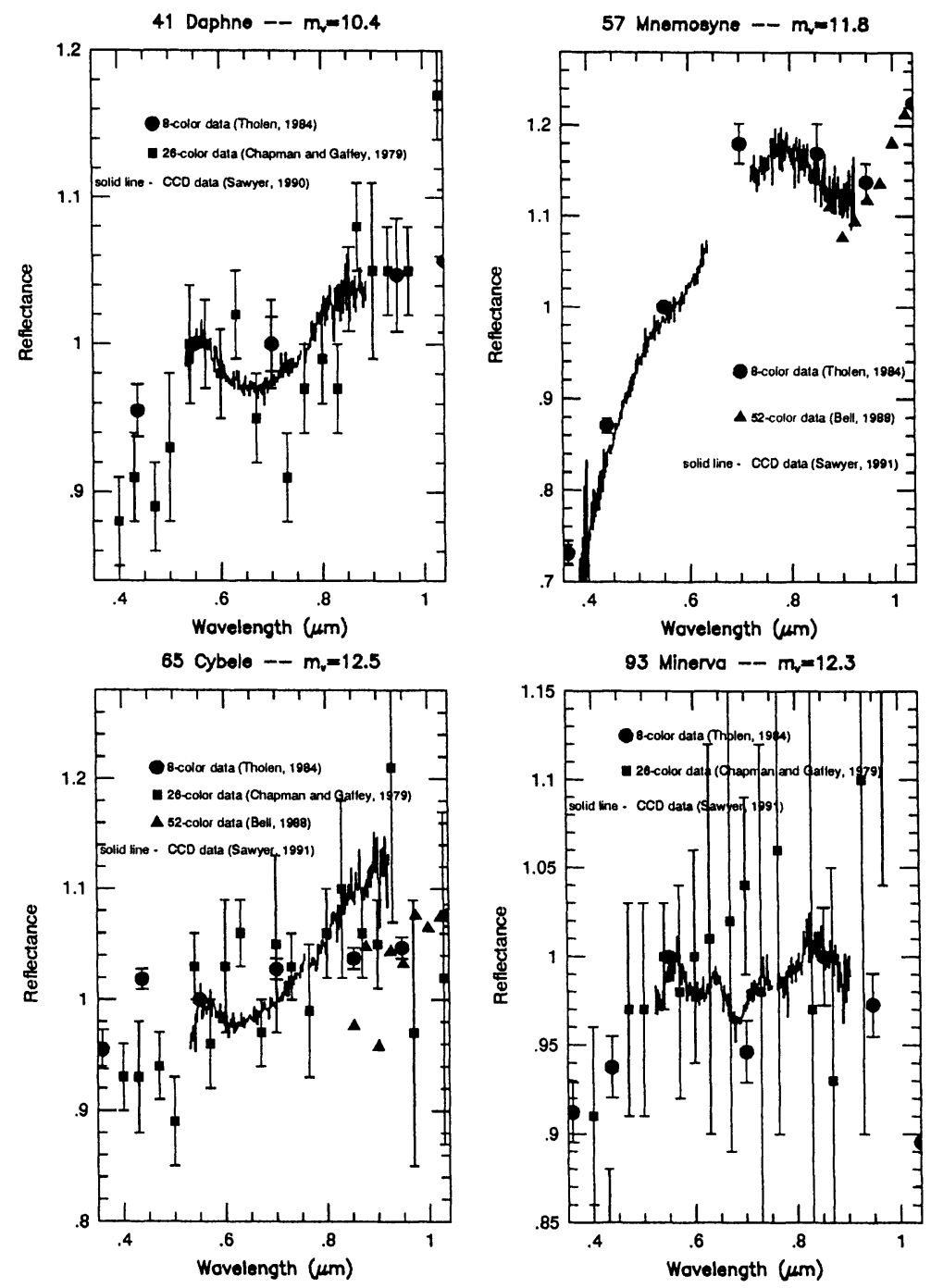

Fig. 2. Comparison of ECAS, 26-color and 52-color survey observations of asteroids and CCD spectra. The CCD observations are of four asteroids from Sawyer (1991). These objects are of a variety of taxonomic types and range in magnitude from 10.4-12.5. Clearly, it is possible to identify more accurately features in the CCD spectra than in the multicolor photometry. 
and LL6 meteorites are the most abundant type of meteorites and, until the Binzel et al. (1993) observation of this asteroid as part of SMASS, no asteroidal analogue had been identified.

\section{COMETS}

The problems of observing comets are often quite different than for other solar system bodies. Though some comets are quite bright, most are quite faint. The nuclei are small (of order a few $\mathrm{km}$ ) and dark (albedo $5 \%$ ). This nucleus is shrouded by a coma and cannot be viewed from earth except when a comet is far from the Sun, when it is especially faint.

Comets are spatially quite extended objects. Depending on the comet and gecrentric distance, cometary comae can subtend several arcmins, while tails can be a degree or longer.

The brightest comets are those which are active near the Sun. Generally, this means that they are brightest at small solar elongation angles and must be observed at large airmass or even during twilight. The orbits can have any inclination, resulting in short windows-of-opportunity for some objects and offering challenges to tracking.

In the absence of chemical reactions, material flows outward from the nucleus as $1 / \mathrm{r}^{2}$, where $r$ is the distance to the nucleus. Photochemical reactions destroy species faster. Thus, the outer regions of the coma are substantially fainter than the optocenter, requiring dynamic ranges to be two or more orders of magnitude.

Fig. 3 illustrates the changes in cometary spectroscopy which are achieved in the transition from non-array instruments to array instruments. The left-hand panels in this figure show data obtained with an Intensified Dissector Scanner (IDS) spectrograph. The right-hand panels show data obtained with a long slit spectrograph imaging onto a CCD detector. All observations were obtained with the McDonald Observatory 2.7-m telescope and are of comet Schaumasse.

The IDS was a dual beam spectrograph which obtained two simultaneous spectra 52 arcsec apart. Beam switching was used to alternately image object and sky down each entrance aperture. The telescope was beam switched every 50 seconds and many such observations summed to form the resultant spectra. This cycle yielded two spectra on the optocenter (one through each aperture) and one spectrum each 52 arcsec either side of the optocenter. Of course, there was an overhead to perform the beam switching. The top, left panel shows one of the spectra of the optocenter imaged through a $4 \times 4 \operatorname{arcsec}^{2}$ slit. The total exposure time was $4800 \mathrm{sec}$ but the elapsed time was $6540 \mathrm{sec}$. At the same time as the two optocenter spectra were obtained, the two spectra 52 arcsec away were obtained, one of which is shown in the middle panel. The four resultant column densities are shown in the lower panel.

The long slit CCD spectrograph has a slit which is $\sim 150$ arcsec long with each pixel being $1.3 \mathrm{arcsec}$ in the spatial direction. We used a slit width of two arcsec. Thus, the CCD effective aperture for each pixel is $1.3 \times 2 \operatorname{arcsec}^{2}$. We exposed for $3600 \mathrm{sec}$. There is no beam switching overhead so the exposure time and the elapsed time were the same. The top panel shows the spectrum from the optocenter. It looks quite similar to the IDS spectrum in the wavelength overlap region, except that it is clearly slightly higher signal/noise even though the exposure time and elapsed time for the CCD image was much shorter. The middle panel shows the spectrum 52 arcsec from the optocenter, comparable to the IDS "off" position. Clearly, this 


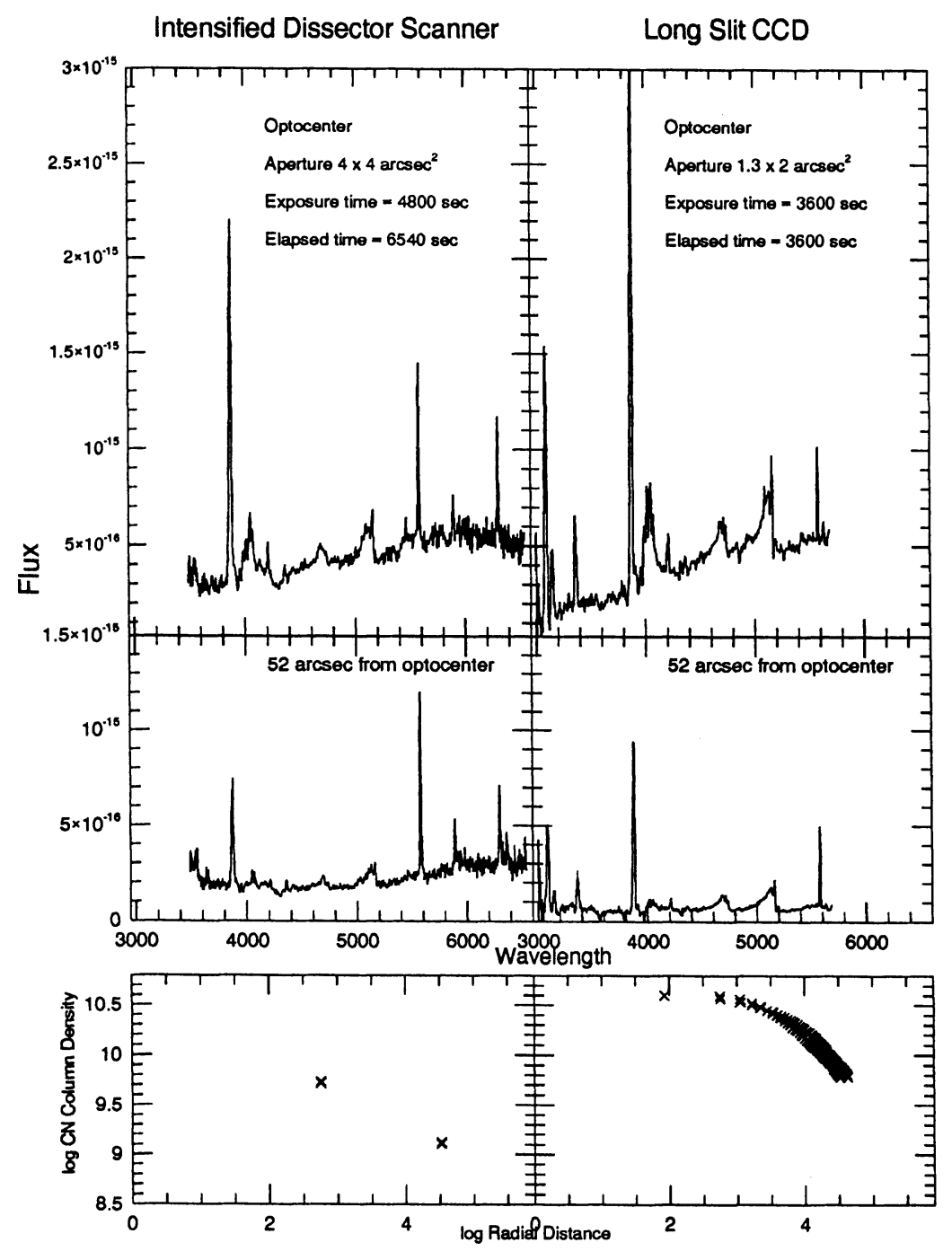

Fig. 3. Comparison of Intensified Dissector Scanner spectra and CCD long slit spectra. The IDS spectral information is the left-hand panels and the CCD is the right-hand panels. From top to bottom, we show optocenter spectra, spectra 52 arcsec from the optocenter and column densities measured. See text for more information. 
spectrum is much higher signal/noise than the comparable IDS spectrum. The bottom panel shows the real strength of the CCD for cometary spectroscopy. Instead of four column density points in $6540 \mathrm{sec}$ elapsed time, a single spectral image of $3600 \mathrm{sec}$ yields 120 column densities. It is obvious that one can define well the gas distribution profile from the CCD data but not from the IDS data.

Since we believe that comets are remnants from the early solar nebula which have undergone very little change since their time of formation, understanding the isotopic abundances of their constituents is important to provide constraints to solar nebula models. Unfortunately, isotopic studies are quite difficult since even the brightest comets have relatively low surface brightnesses.

One of the nicest examples of isotopic studies of comets is that of Kleine (1994; Kleine et al. 1994, 1995a, b). Using the Photon Counting Array on the coudé spectrograph ( 75,000 ) at the Mt. Stromlo 74-inch reflector, this group observed one periodic comet (Halley) and three long-period or non-periodic comets (Levy, Austin and Okazaki-Levy-Rudenko) to determine the ${ }^{12} \mathrm{C} /{ }^{13} \mathrm{C}$ abundance via the R-branch of the $\mathrm{B}^{2} \Sigma^{+}-\mathrm{X}^{2} \Sigma^{+}$band of $\mathrm{CN}$. The carbon isotope ratios determined were between 85 and 95, indicating that the ${ }^{13} \mathrm{CN}$ lines are much weaker than the ${ }^{12} \mathrm{CN}$ lines. To determine the isotopic ratio, Kleine (1994) and Kleine et al. (1994, 1995a, b) had to model the complete P, Q and R branch of CN to predict the position and strength of the isotopic lines. The overlap of the various branches makes it easy to identify the wrong weak lines as isotopic lines. Fig. 2.14 of Kleine (1994) shows the model and the Halley data illustrating the problem of weak P-branch lines in the region of the strong R-branch lines. In addition, the Swings effect changes the ratio of strengths of the various lines (see Fig. 2.1 of Kleine (1994)).

Once the data were modeled, Kleine (1994) was able to identify and measure the various lines present. This resulted in the identification of some very weak ${ }^{13} \mathrm{CN}$ lines on the wings of stronger ${ }^{12} \mathrm{CN}$ lines of the R-branch. Fig. $4.11 \mathrm{~b}$ of Kleine (1994) shows the $\mathrm{R}(0)$ through $\mathrm{R}(7)$ lines and the accompanying isotopic lines. The isotopic abundances which were determined in this manner are quite important for determining if comets have interstellar ${ }^{12} \mathrm{CN} /{ }^{13} \mathrm{CN}$ ratios of a few or solar system values around 90 . The results of this study of four comets shows that comets have solar system ${ }^{12} \mathrm{CN} /{ }^{13} \mathrm{CN}$ ratios, and that this ratio is consistent from comet-to-comet.

Another nice feature of the observations of Kleine (1994) and Kleine et al. (1994, 1995a, b) is that the Mt. Stromlo coudé spectrograph employs a long slit. Thus, not only was it possible to determine the isotopic abundance of carbon, but it was possible to study the spatial distribution of the gas at high resolution. Fig. 2.6 of Kleine (1994) shows an example of spatial information for Halley. The data are scaled to the $\mathrm{R}(8)$ line (the strongest) and the figure shows that the ratios of the strengths of various $\mathrm{R}$-branch lines changes with position. This is the Greenstein effect, or differential Swings effect, and has important uses for determining outflow velocities of the coma.

\section{SUMMARY}

The examples given in this paper are but a few of the ways in which modern array detectors have increased the scientific information on solar system bodies. Good quantum 
efficiency has allowed studies of asteroids and comets to push to fainter limits. Spectral coverage has allowed for in-depth, high-spectral-resolution coverage of brighter objects. High signal/noise has allowed very weak features to be detected for the first time. Large dynamic range has allowed for searches for very weak features, such as lunar sodium and potassium, near a bright source. The two dimensional format of arrays has allowed for spatial coverage well suited to the extended nature of solar system bodies such as Jupiter and comets. The nature of array detectors not only improves upon the types of data which has been collected in the past, but allows for new types of data, new questions, and new insights in our study of the origin and evolution of the solar system.

\section{ACKNOWLEDGEMENTS}

I thank all the planetary scientists who supplied figures for my presentation at the IAU symposium and who allowed me to reference work in progress. In particular, thanks are due to Drs. R. Binzel, W. Cochran, B. Flynn, S. Sawyer, J. Spencer, P. Wehinger and to their collaborators on these data.

\section{REFERENCES}

Bell, J. F., Hawke, B. R., Owensby, P. D., and Gaffey, M. J. 1988 Lunar Planet. Sci. Conf. XIX, 57

Binzel, R. P. and Xu, S. 1993 Science 260, 186

Binzel, R. P., Xu, S., Bus, S. J., Skrutskie, M. F., Meyer, M. R., Knezek, P. and Barker, E. S. 1993 Science 262, 1541

Chapman, C. R. and Gaffey, M. J. 1979 in Asteroids, T. Gehrels, ed., The University of Arizona Press, Tucson, p. 1064

Chapman, C. R., Morrison, D. and Zellner, B. 1975 Icarus 25, 104

Kleine, M. 1994 Carbon Isotope Abundance Ratios in Comets, Ph.D. thesis, Arizona State University.

Kleine, M., Wyckoff, S., Wehinger, P. A. and Peterson, B. A. 1994 ApJ 436, 885

Kleine, M., Wyckoff, S., Wehinger, P. A. and Peterson, B. A. 1995a ApJ 439, 1021

Kleine, M., Wyckoff, S., Wehinger, P. A. and Peterson, B. A. 1995b ApJ 439, 1021

McFadden, L. A., Bell, J. F. and McCord, T. B. 1980 Icarus 44, 410

Potter, A. E. and Morgan, T. H. 1988 Science 241, 675

Sawyer, S. R. 1991 Ph.D. thesis, The University of Texas at Austin

Spencer, J. R., Calvin, W. M. and Person, M. J. 1994 JGR Planets, submitted

Tholen, D. J. 1984 Ph.D. thesis, Univ. of Arizona

Vilas, F. and McFadden, L. A. 1992 Icarus 100, 85

Woodman, J. H., Cochran, W. D. and Slavsky, D. B. 1979 Icarus 37, 73

Xu, S., Binzel, R. P., Burbine, T. H. and Bus, S. J. 1994 Submitted to Icarus 


\section{DISCUSSION}

WEST: A very important effect of the new detector technologies for solar system research, not mentioned by the speaker, is the probability to observe extremely faint and distant, minor objects, e.g. the new trans-Neptunian objects and long period comets on their way out. This has in fact opened up an entirely new field in the solar system research.

COCHRAN: I agree entirely. However, most of these new objects are too faint to be observed spectroscopically, at least with today's technology. This talk concentrated entirely on spectroscopic applications of arrays, but imaging applications are certainly opening up new opportunities.

WATSON: Are the terrestrial $\mathrm{NaD}$ emission lines present in the high resolution spectrum of the linear atmosphere you showed?

COCHRAN: No, the terrestrial emission lines are too faint to show up. Flynn and Stern moved the telescope back to the same region of the sky in which they had observed the moon and obtained a comparable length exposure of the sky. At this resolution and the short exposure times, no terrestrial $\mathrm{NaD}$ is observed. 